Proyecciones Journal of Mathematics

Vol. 40, No 6, pp. 1641-1655, December 2021.

Universidad Católica del Norte

Antofagasta - Chile

\title{
Power domination in splitting and degree splitting graph
}

\author{
J. Anitha \\ Arulmigu Kapaleeswarar Arts and Science College, India \\ and \\ S. Muthukumar \\ Easwari Engineering College, India \\ Received : December 2020. Accepted : July 2021
}

\begin{abstract}
$A$ vertex set $S$ is called a power dominating set of a graph $G$ if every vertex within the system is monitored by the set $S$ following a collection of rules for power grid monitoring. The power domination number of $G$ is the order of a minimal power dominating set of $G$. In this paper, we solve the power domination number for splitting and degree splitting graph.
\end{abstract}

Keywords: Power dominating set; Electrical power network; splitting graph; degree splitting graph. 


\section{Introduction}

A graph $G=(V, E)$ is an ordered pair of a finite nonempty set of vertices $V=V(G)$ and a set of edges $E=E(G)$ consists of unordered pairs of different vertices. The order of $G$ is denoted by $|G|=|V(G)|$. We are saying that the vertices $u$ and $v$ are adjacent, if $u v \in E$. For $v \in V(G)$. The open neighbourhood of $v$, denoted by $N(v)$, is the set of vertices adjacent to $v$; the closed neighbourhood of $v$, denoted by $N[v]$, is $N_{G}(v) \cup\{v\}$. Let $S \subseteq V(G)$. Then open neighbourhood of $S$ is defined as $N(S)=\bigcup_{v \in S} N(v)$ and the closed neighbourhood of $S$ is defined as $N[S]=N(S) \cup S[3]$.

For a graph $G(V, E), S \subseteq V$ is a dominating set of $G$ if every vertex in $V \backslash S$ has at least one neighbour in $S$. The domination number of $G$, denoted by $\gamma(G)$, is the minimum cardinality of a dominating set of $G$. [3].

For a graph $G$, let $S \subseteq V(G)$. We define the sets $M^{j}(S)$ of vertices monitored by $S$ at level $j, j \geq 0$, inductively as follows:

1. $M^{0}(S)=N[S]$.

2. $M^{j+1}(S)=M^{j}(S) \cup\left\{w: \exists v \in M^{j}(S), N(v) \cap\left(V(G) \backslash M^{j}(S)\right)=w\right\}$.

If $M^{\infty}(S)=V(G)$, then the set $S$ is said to be a power dominating set of $G$. The minimum number of a power dominating set in $G$ is called the power domination number of $G$ and is denoted by $\gamma_{p}(G)$ [3].

The power domination problem is $N P$-complete [1]. The problem has been exhibited to be $N P$-complete even when restricted to bipartite graphs and chordal graphs [1]. The bounds on the power domination number for any graph $G, 1 \leq \gamma_{p}(G) \leq \gamma(G)$, were realized in [1] and also power domination problem is well investigated for trees [1]. In 2005 Liao et al. have obtained that the power domination number for split graphs, a subclass of chordal graphs, an NP-complete. The power domination problem was solved for block graphs [4], product graphs [5], cylinder, torus and generalized Petersen graphs [6], certain chemical structures [7], honeycomb network [3], hexagonal grid [8] and so on.

- The central graph of $G$, written as $C(G)$ is obtained by subdividing each edge of $G$ exactly once and joining all the non-adjacent vertices of $G$ in $C(G)[9]$.

- Let $v \in G$ and let $v^{\prime}$ be a new vertex. Join $v^{\prime}$ to all vertices of $G$ adjacent to $v$. The graph $S(G)$ thus obtained is named thesplitting 
of a graph $G$. Let $G$ be a graph with $V=D_{1} \cup D_{2} \ldots \cup D_{r} \cup L$ where each $D_{i}$ may be a set of vertices having a minimum of two vertices and having the identical degree and $L=V-\cup D_{i}, 1 \leq i \leq r$. The degree splitting graph of $G$, denoted by $D S(G)$ is obtained from $G$ by adding vertices $w_{1}, w_{2}, \ldots, w_{r}$ and joining $w_{i}$ to every vertex of $D_{i}$ [11].

- Let $T$ be the tree constructed from a $K_{1, n}$ by subdividing any number of its edges any number of times; that is, $T$ has at the most one vertex of degree 3 or more. We call such a tree $T$ a spider, denoted by $S(n)$ [1]. A tree is a connected graph that contains no cycles. The most common type of tree is the binary tree. It is so called because each node can have at most two descendants. A $k$-ary tree is said to be a complete $k$-ary tree if each internal node has exactly $k$ - descendants[1].

- The friendship graph $F_{m}$ may be formulated by joining $m$ copies of the cycle graph $C_{m}$ with a typical vertex. A wheel graph $W_{m}$ of order $m$, called an $m$-wheel is, a graph that contains a cycle of order $m-1$, and for which every graph vertex within the cycle is connected to at least one other graph vertex (which is understood because hub). The edges of a wheel which include the hub are called spokes. The helm graph $H_{m}$ is the graph obtained from an $m$-wheel graph by adjoining a pendant edge at every vertex of the cycle [10].

- The spiked cycle with $2 n$ vertices, denoted as $S C_{n}$, is that the graph with vertex set $\left\{v_{0}, \ldots, v_{n-1}\right\} \cup\left\{u_{0}, \ldots, u_{n-1}\right\}$ and edges $v_{i} v_{i+1}$ and $v_{i} u_{i}$, for all $i=0, \ldots, n-1$ with the subscript addition modulo $n$. It is observed that the vertices $\left\{v_{0}, \ldots, v_{n-1}\right\}$ are cyclically connected by the edges $v_{i} v_{i+1}$ while the edges $v_{i} u_{i}$ add a spoke on every vertex of the cycle [6].

- The Cartesian product $G H$ of two graphs $G$ and $H$ is the graph with vertex set $V(G) \times V(H)$, two vertices $(r, t)$ and $(s, p)$ are adjacent if and only if either $r=s$ and $t p \in E(H)$ or $t=p$ and $r s \in E(G)$. The $n$-ladder graph $L$ of length $n$ is defined as $P_{2} P_{n+1}$, where $P_{n+1}$ may be a path on $n+1$ vertices, $n \geq 1$. The circular ladder $C L_{n}$ of length $n \geq 3$ is that the Cartesian product $C L_{n}=C_{n} K_{2}$. Möbius Ladder graphs are formulated by introducing a twist in a circular ladder and is denoted by $M_{n}$ [14]. 
- The graph $P_{m} P_{n}$ is termed $s \times r$ grid graph, and is denoted by $M(m, n), m, n \geq 2$. The cylinder $C_{m} P_{n}$, where $m, n \geq 3$ is a $P_{m} P_{n}$ grid with wraparound edge in each row. It is observed that the vertex set of $P_{m} \times P_{n}$ is $V=\left\{u_{1} u_{2}: 0 \leq u_{i} \leq d_{i-1}, i=1,2\right\}$ and two vertices $u=u_{1} u_{2}$ and $v=v_{1} v_{2}$ are linked by an edge, if $\left|u_{1}-v_{1}\right|+\left|u_{2}-v_{2}\right|=1$ $[13]$.

\section{Main Results}

In this section, we solve the power domination number for central graph, splitting graph and degree splitting graph for extended cycle of ladder, circular ladder and corona graph.

\section{Observations I:}

The following results hold good:

a. For any graph $G$ of order $r \geq 2, \gamma_{p}(C(G))=1$.

b. For any graph $G, 1 \leq \gamma_{p}(S(G)) \leq \gamma(G)$.

c. Let $G$ be a graph. Then $\gamma_{p}(S(G))=1$, where $G \in\left\{P_{n}, K_{n}, F_{m}, S(n), W_{m}\right\}$. See Figure 1 $(a)$.

Let $G$ be a cycle $C_{n}, n \geq 3$. Then $\gamma_{p}(S(G))=2$.

Proof. Let $v_{1}, v_{2}, \ldots, v_{n}$ be the vertices of $C_{n}$ which are replicated by the vertices $v_{1}^{\prime}, v_{2}^{\prime}, \ldots, v_{n}^{\prime}$ and let $e_{1}, e_{2}, \ldots, e_{n}$ be the edges of $C_{n}$. Then the graph $S\left(C_{n}\right)$ will have $2 n$ vertices and $3 n$ edges.

Let $S$ be a power dominating set of $S\left(C_{n}\right)$. We claim that $|S|=2$.

( $\geq$ :)Suppose $|S|=1$. Without loss of generality, let $S=\left\{v_{1}\right\}$ be the power dominating set of $S\left(C_{n)}\right.$. Then $M^{0}(S)$ monitors the vertices say, $\left\{v_{1}, v_{2}, v_{n}, v_{2}^{\prime}, v_{n}^{\prime}\right\}$. See Figure $1(c)$. Now the vertices in $M^{0}(S)$ say, $v_{2}^{\prime}$ and $v_{n}^{\prime}$ are adjacent to exactly one unmonitored vertices say, $v_{3}$ and $v_{n-1}$ respectively. Then these vertices are monitored in $M^{1}(S)$. Now for every vertex $u \in M^{1}(S),\left|N[u] \backslash M^{1}(S)\right| \geq 2$, a contradiction. See Figure $1(c)$.

$(\leq:)$ Select the vertices $\left\{v_{1}, v_{5}\right\}$ in $S$. Then $M^{0}(S)=\left\{v_{1}, v_{n}, v_{2}, v_{2}^{\prime}, v_{n}^{\prime}, v_{5}, v_{4}, v_{6}, v_{4}^{\prime}, v_{6}^{\prime}\right\}$. In the next step vertices labelled as $v_{2}^{\prime}, v_{6}^{\prime}$ and $v_{n}^{\prime}$ in $M^{0}(S)$ is adjacent to exactly one unmonitored vertices say, $v_{3}, v_{7}$ and $v_{n-1}$ respectively, these vertices can monitor in $M^{1}(S)$. Proceeding inductively, for every vertex $u \in M^{i}(S),\left|N[u] \backslash M^{i}(S)\right|=1$, at every inductive step $i \geq 2$. Thus $M^{n-1}(S)=V(G)$. Hence $|S|=2$. Therefore, $\gamma_{p}(G)=2$. 


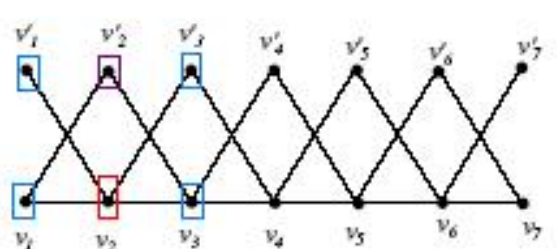

(a)

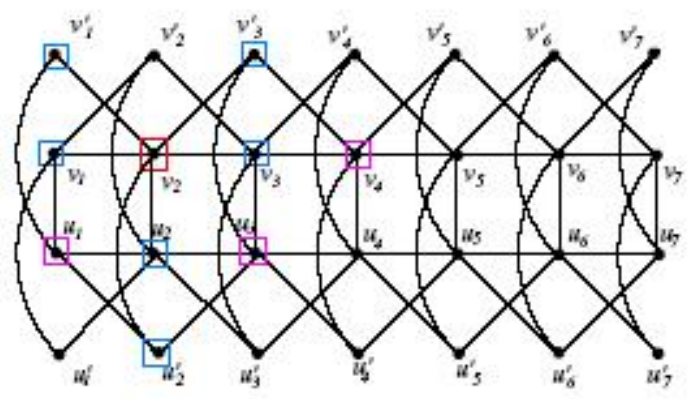

(b)

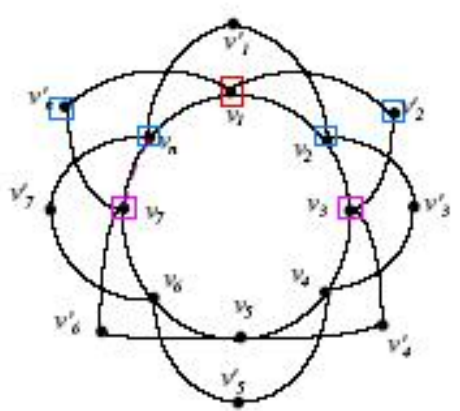

(c)

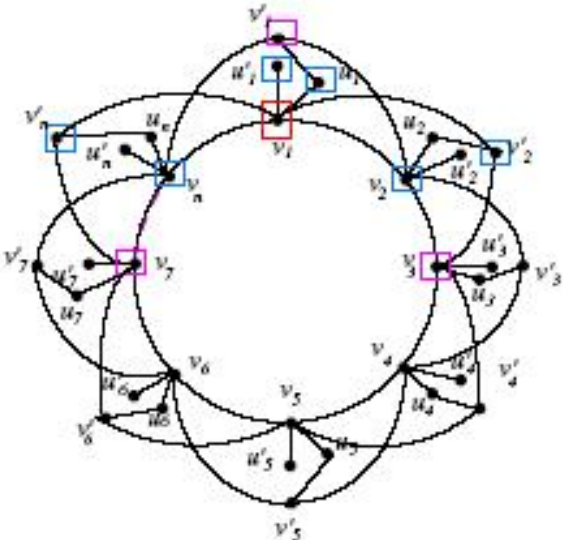

(d)

Figure 2.1: Red colored squared vertices indicates a minimum power dominating set of (a)Path $S\left(P_{7}\right)$ (b) Ladder $S\left(L_{7}\right) \quad$ (c)Cycle $S\left(C_{7}\right) \quad$ (d) Spiked Cycle $S\left(S C_{7}\right)$.

\subsection{Graphs with $\gamma_{p}(S(G))=\gamma_{p}(G)$}

In this section, we realize graphs for which $\gamma_{p}(S(G))=\gamma_{p}(G)$, where $G$ is isomorphic to complete bipartite graph, spiked cycle and circular ladder .

Let $G$ be a spiked cycle $S\left(S C_{n}\right), n \geq 3$. Then $\gamma_{p}(G)=\left\lceil\frac{n}{3}\right\rceil$.

Proof. Label the cyclically connected vertices of $S C_{n}$ as $v_{0}, v_{2}, \ldots, v_{n}$ which are replicated by the vertices $v_{0}^{\prime}, v_{2}^{\prime}, \ldots, v_{n}^{\prime}$ and let $u_{0}, u_{2}, \ldots, u_{n}$ be the spoke vertices of $S C_{n}$ which are replicated by the vertices $u_{0}^{\prime}, u_{2}^{\prime}, \ldots, u_{n}^{\prime}$. Then the graph $S\left(S C_{n}\right)$ will have $4 n$ vertices. 
( $\leq$ :) Let $S$ be a power dominating set of $S\left(S C_{n}\right)$. Let $S=\left\{v_{i}: i \equiv\right.$ $0 \bmod 3\}$ where $1 \leq i \leq n$ with $|S|=\left\lceil\frac{n}{3}\right\rceil$. Then

$M^{0}(S)=\left\{v_{i}, u_{i}, u_{i}^{\prime}, v_{i+1}, v_{i-1}, v_{i+1}^{\prime}, v_{i-1}^{\prime}: i \equiv 0 \bmod 3\right\}$. Now the vertices labelled as $\left\{v_{i+1}^{\prime}, v_{i-1}^{\prime}\right\}$ in $M^{0}(S)$ are adjacent to exactly one unmonitored vertices say, $\left\{u_{i+1}, u_{i-1}\right\}$. These vertices monitored in $M^{1}(S)$. In the next step, vertices lablled as $\left\{v_{i+1}, v_{i-1}\right\}$ are adjacent to exactly one unmonitored vertices say, $\left\{u_{i+1}^{\prime}, u_{i-1}^{\prime}\right\}$ and these vertices monitored in $M^{2}(S)$. Hence $M^{2}(S)=V\left(S\left(S C_{n}\right)\right)$. This implies that, $\gamma_{p}\left(S\left(S C_{n}\right)\right) \leq\left\lceil\frac{n}{3}\right\rceil$.

$(\geq:)$ As $\Delta\left(S\left(S C_{n}\right)\right)=6$, we notice that $\operatorname{deg}(v)=6$ for every $v \in S$. In particular, every vertex in $S$ lies on the cycle $\left(v_{1}, v_{2}, \ldots, v_{n}\right)$ of $S\left(S C_{n}\right)$. Suppose that $\gamma_{p}\left(S\left(S C_{n}\right)\right)<\left\lceil\frac{n}{3}\right\rceil$. Then there is a vertex say, $v_{i} \in S\left(S C_{n}\right)$ such that $v_{i} \notin S$ and $d\left(v_{i}, N(s)\right)=1$. So $v_{i} \notin N[S]$. This vertex can be monitor only if at least one edge incident to it is monitored. The edge $u_{i} v_{i}$ and $u_{i}^{\prime} v_{i}$ cannot be monitor unless if $v_{i-1} v_{i}, v_{i+1} v_{i}, v_{i-1} v_{i}^{\prime}$ and $v_{i-1} v_{i}^{\prime}$ are monitored. So $v_{i-1} v_{i}$ is also not monitored. See Figure $1(d)$. Hence $S$ is not a power dominating set, a contradiction. Therefore $\gamma_{p}(G) \geq\left\lceil\frac{n}{3}\right\rceil$. Hence the proof. 2 .

Let $G$ be a ladder $L_{n}$ or circular ladder $C L_{n}, n \geq 4$, we have $\gamma_{p}(S(G)) \geq$

Proof. Let $S$ be a power dominating set of $S\left(C L_{r}\right)$. We claim that $|S| \geq 2$. Suppose not, consider $|S|=1$. Without loss of generality, let $S=$ $\{w\}$. Then for every vertex in $u \in N(w)$ is adjacent to two neighbouring vertices, a contradiction. See Figure $1(b)$.

Let $G$ be a circular ladder $C L_{n}, n \geq 4$ or a Möbius ladder $M_{n}, n \geq 4$. Then $\gamma_{p}(S(G))=2$.

Proof. Let $v_{1}, v_{2}, \ldots, v_{n}$ and $u_{1}, u_{2}, \ldots, u_{n}$ be the vertices of $C L(n)$ which are replicated by the vertices $v_{1}^{\prime}, v_{2}^{\prime}, \ldots, v_{n^{\prime}}$ and $u_{1}^{\prime}, u_{2}^{\prime}, \ldots, u_{n}^{\prime}$. Then the graph $S(C L(n))$ will have $4 n$ vertices. Select the vertices $\left\{v_{1}, v_{4}\right\}$ in $S$ as shown in Figure $2(a)$. Then $M^{0}(S)=\left\{v_{1}, v_{n}, v_{2}, v_{2}^{\prime}, v_{n}^{\prime}, u_{1}^{\prime}, u_{1}\right.$, $\left.v_{4}, v_{3}, v_{5}, v_{3}^{\prime}, v_{5}^{\prime}, u_{4}^{\prime}, u_{4}\right\}$. In the next step vertices say, $v_{2}^{\prime}$ and $v_{3}^{\prime}$ in $M^{0}(S)$ are adjacent to exactly one unmonitored vertices say, $u_{3}$ and $u_{2}$ respectively, these vertices can be monitored in $M^{1}(S)$. Then vertices labelled as $v_{3}$ and $v_{2}$ in $M^{0}(S)$ monitor $u_{3}^{\prime}$ and $u_{2}^{\prime}$ in $M^{2}(S)$. Proceeding inductively, for every vertex $u \in M^{i}(S),\left|N[u] \backslash M^{i}(S)\right| \leq 1, i \geq 1$. Thus $M^{n-1}(S)=V(G)$. Hence $|S|=2$. Therefore, $\gamma_{p}(G)=2$.

Observation 1. Let $G$ be a Hexagonal chain $H_{n}, n \geq 3$. 
Then $\gamma_{p}(S(G))=2$.

Observation 2. For the complete bipartite graph $K_{m, n}, m, n \geq 3$, we have $\gamma_{p}\left(S\left(K_{m, n}\right)\right)=2$.

\subsection{Extended Cycle-of-Ladder}

Jywe-Fei Fang proposed a network called cycle-of-ladder in 2008. It is a spanning subgraph of the hypercube network, thereby showing that hypercube network is bipancyclic [14]. The graph obtained appears like a ladder having two rails and $n+1$ rungs between them. The length of the ladder is defined as $n$.

[14] A cycle-of-ladder is a graph, composed of a cycle $C_{s}$ of length $2 l$ termed the spine cycle such that removal of alternate edges on $C_{s}$ leaves $l$ distinct components $L_{1}, L_{2}, \ldots, L_{l}$, each of which is isomorphic to a ladder. If $r_{1}, r_{2}, \ldots, r_{l}$ represents the number of rungs in the ladders $L_{1}, L_{2}, \ldots, L_{l}$ respectively, then cycle of ladders is written as $C L\left(2 l, r_{1}, r_{2}, \ldots, r_{k}\right)$. Let $R_{j}^{i}, 1 \leq j \leq r_{i}$ represent the rungs of $L_{i}$ such that the bottom rung $R_{1}^{i}$ is the edge of $C_{s}$ in $L_{i}, 1 \leq i \leq k$. For brevity, we represent $\left(r_{1}, r_{2}, \ldots, r_{k}\right)$ as $s$ and we represent the cycle-of-ladder as $C L(2 l, s)$, where $l$ and $s$ denote the number of ladders and the length of each ladder respectively.

For convenience, we label the vertices of $L_{i}$ as $l_{j, 1}^{i}$ and $l_{j, 2}^{i}$ where $0 \leq j \leq s$ and $1 \leq i \leq l$ in $C L(2 l, s)$.

We add $l$ number of edges to $C L(2 l, s)$ to obtain a 3-regular graph and call it the extended cycle-of-ladder $E C L(2 l, s)$.

[14] The extended cycle-of-ladder $E C L(2 l, s)$ is obtained from $C L(2 l, s)$ by adding edges between $\left(l_{s, 2}^{i}, l_{s, 1}^{i+1}\right), 1 \leq i \leq l-1$ and $l_{s, 2}^{l}, l_{s, 1}^{1}$. 


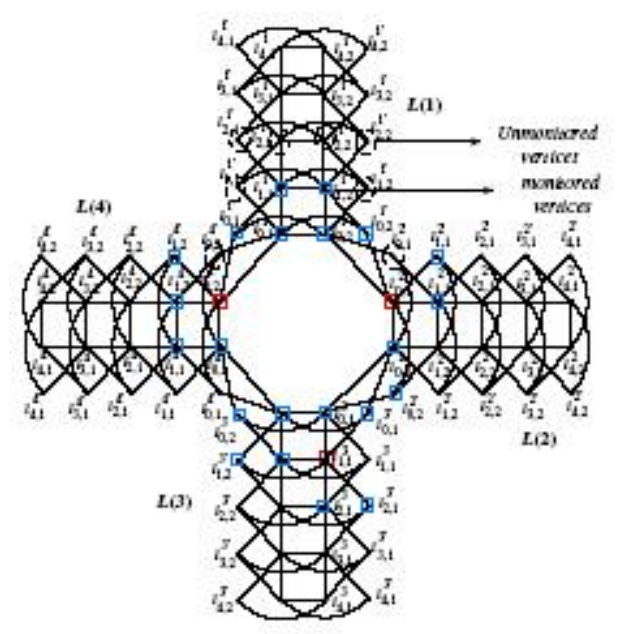

(ब)

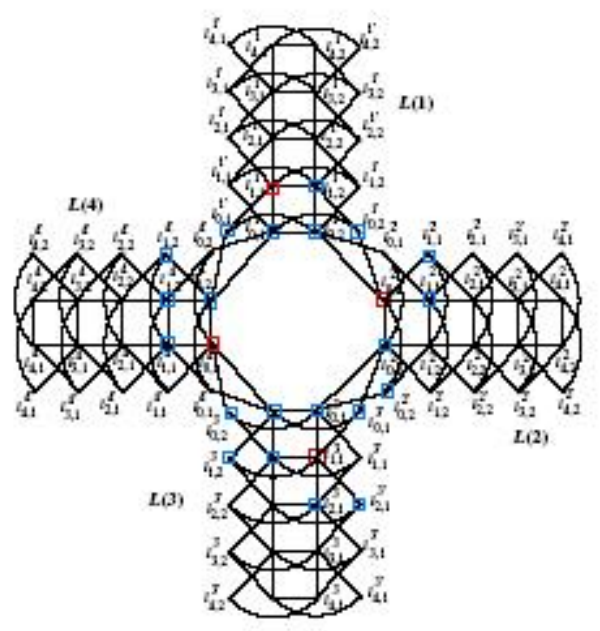

(b)

Figure 2.2: Red colored squared vertices indicates a power dominating set of $(a) C L(8,5)$ and $(b) C L(8,5)$.

Let $G$ be an extended cycle-of-ladder $\operatorname{ECL}(2 l, s), l, s \geq 4$. Then $\gamma_{p}(S(G)) \geq l$.

Proof. $\quad C L(2 l, s)$ contains $l$ vertex disjoint copies of $L(i)$, say

$L(1), L(2), \ldots, L(l)$. Let $S$ be a power dominating set of $E C L(2 l, s)$. We claim that $|S| \geq l$. Suppose $|S|=l-1$. Without loss of generality, consider $L(1)$. The worst case arises when all bone cycle vertices and their duplicated vertices $l_{0,1}^{1}, l_{0,2}^{1}, l_{0,1}^{1}{ }^{\prime}, l_{0,2}^{1}{ }^{\prime}$ of $L(1)$ are monitored by vertices of $S$ not in $L(1)$. Then the vertices $l_{0,1}^{1}, l_{0,2}^{1}$ are adjacent to two unmonitored vertices each say, $l_{1,1}{ }^{\prime}, l_{0,2}{ }^{\prime}{ }^{\prime}$ and $l_{1,2}{ }^{\prime}, l_{0,1}{ }^{\prime}$ respectively and $l_{0,1}^{1}{ }^{\prime}, l_{0,2}{ }^{\prime}$ are adjacent to exactly one unmonitored vertices say, $l_{1,1}^{1}, l_{1,2}^{1}$ each of which in turn is adjacent to at least two unmonitred vertices, a contradiction. Therefore, $|S| \geq l$. See Figure 2(a). 


\section{Algorithm for Power Domination in Splitting graph of Cycle-of- Ladder $S(C L(2 l, s))$}

Input: Splitting graph of Cycle-of-ladder $S(C L(2 l, s)), l, s \geq 4$.

Algorithm: Label the vertices of splitting graph of cycle-of-ladder as $\left\{1_{j, 1}^{i} \cup l_{j, 2}^{i} \cup\left(l_{j, 1}^{i}\right)^{\prime} \cup\left(l_{j, 2}^{i}\right)^{\prime}: 0 \leq j \leq s, 1 \leq i \leq l\right\}$ and select the vertices $\left\{l_{1,1}^{i}, l_{0,1}^{j}: i=1,3,5, \ldots, l-1, j=2,4,6, \ldots, l\right\}$ in $S$. See Figure $2(b)$.

Output: $\gamma_{p}(S(C L(2 l, s))=l$.

Proof of Correctness: Let $S$ be a power dominating set of $S(E C L(2 l, s))$. Now vertices in $S$ monitor all the vertices of bone cycle and the replicated vertices of bone cycle say, $l_{0,1}^{1}, l_{0,2}^{1}, l_{0,1}^{1}{ }^{\prime}, l_{0,2}^{1}{ }^{\prime}, \ldots, l_{0,1}^{l}, l_{0,2}^{l}, l_{0,1}{ }^{\prime}{ }^{\prime}, l_{0,2}{ }^{\prime}$. Then the vertices labelled as $l_{0,1}^{1}{ }^{\prime}, l_{0,2}^{1}{ }^{\prime}, \ldots, l_{0,1}^{l}{ }^{\prime}, l_{0,2}^{l}{ }^{\prime}$ are adjacent to exactly one unmonitored vertices say, $l_{1,1}^{1}, l_{1,2}^{1}, \ldots, l_{1,1}^{l}, l_{1,2}^{l}$ these vertices monitored in $M^{1}(S)$. In the next step, the vertices labelled as $l_{0,1}^{1}, l_{0,2}^{1}, \ldots, l_{0,1}^{l}, l_{0,2}^{l}$ are adjacent to exactly one unmonitored vertices say, $l_{1,1}^{1}{ }^{\prime}, l_{1,2}^{1}{ }^{\prime}, \ldots, l_{1,1}{ }^{\prime}, l_{1,2}{ }^{\prime}$ these vertices monitored in $M^{2}(S)$. Now the vertices labelled as $l_{1,1}^{1}{ }^{\prime}, l_{1,2}^{1}{ }^{\prime}, \ldots, l_{1,1}{ }^{\prime}, l_{1,2}{ }^{\prime}{ }^{\prime}$ are adjacent to exactly one unmonitored vertices say, $l_{2,1}^{1}, l_{2,2}^{1}, \ldots, l_{2,1}^{l}, l_{2,2}^{l}$ these vertices monitored in $M^{3}(S)$. Proceeding inductively, for every vertex $u \in M^{i}(S),\left|N[u] \backslash M^{i}(S)\right| \leq 1, i \geq 4$. Hence $S$ is a power dominating set of $S\left(C L(2 l, s)\right.$ with $|S|=l$. Therefore, $\gamma_{p}(S(C L(2 l, s))=|S|=l$, which we present in the following:

Let $G$ be the graph isomorphic to cycle-of-ladder as $C L(2 l, s), l, s \geq 4$ or an extended cycle-of-ladder $\operatorname{ECL}(2 l, s), l, s \geq 4$. Then $\gamma_{p}(G)=l$

Let $T$ be a complete $k$ - array tree of level $r, r \geq 2, k \geq 2$. Then $\gamma_{p}(S(T))=k^{r-1}$.

Proof. Name the vertices of a complete $k$ - array tree of level $r, r \geq 2$ as $v_{1}, v_{2}, \ldots, v_{n}$ which are replicated by the vertices $v_{1}^{\prime}, v_{2}^{\prime}, \ldots, v_{n}^{\prime}$. Then the splitting graph of $k$-array tree of level $r, r \geq 2$ will have $k^{r+2}-2$ vertices. In $T$, the vertices in level $r-1$ as $2 k$ children adjacent to it. Any minimum power dominating set for a complete $k$-array tree contains 
vertices of level $r-1$. For, if not, even if all vertices in level $(r-1)$ are monitored, their children will be left unmonitored. Select $k^{r-1}$ vertices in level $r-1$ of splitting complete $k$-array tree $S(T)$ in set $S$. The vertices in level $r-1$ monitor $k^{r+1}$ vertices in level $r$ and $k^{r-2}$ vertices in level $r-2$. Now $k^{r}$ vertices in level $r$ monitor $k^{r-1}$ replicated vertices in level $r-1$. Proceeding inductively, then the vertices in level $r-t$ monitor vertices in level $r-t-1,2 \leq t \leq r-1$. Hence $S$ is a power dominating set of $S(T)$ with $|S|=k^{r-1}$. Therefore, $\gamma_{p}(S(T))=|S|=k^{r-1}$.

For the Helm graph $H_{m}, m \geq 3$, we have $\gamma_{p}\left(S\left(H_{m}\right)\right)=m$.

Proof. Label the vertices of helm graph $H_{m}$ as $v_{1}, v_{2}, \ldots, v_{m}$ which are replicated by the vertices $v_{1}^{\prime}, v_{2}^{\prime}, \ldots, v_{m}^{\prime}$. Then the graph $S(G)$ will have $4 m+2$ vertices. Let $S$ be a power dominating set of $S(G)$. Suppose not, by definition there are $m$ vertices of $H_{m}$ in spoke cycle, each of which is adjacent to two pendent vertices in it. Deleting at least one vertex from $S$ we see that at least one vertex of $S\left(H_{m}\right)$ is not monitored by $S$, a contradiction. Thus $|S| \geq m$. Now $S=\left\{v_{1}, v_{2}, \ldots, v_{m}\right\}=V\left(S\left(H_{m}\right)\right)$. Hence $M^{0}(S)=V\left(H_{m}\right)$ is monitored by $S$. Therefore, $\gamma_{p}\left(S\left(H_{m}\right)\right)=m$. Hence the proof.

\subsection{Graphs with $\gamma_{p}(S(G))=\gamma_{p}(G)=\gamma(G)$}

[1] Let $G$ and $H$ be two graphs of order $r$ and $s$ respectively. The corona of $G$ and $H$, denoted by $G \circ H$ is a graph composed from one copy of $G$ and $r$ copies of $H$ where $k^{t h}$ vertex of $G$ is adjacent to every vertex in the $k^{\text {th }}$ copy of $H, 1 \leq k \leq|V(G)|$.

Rajasingh et.al. [12] have proved that domination number and power domination number are equal for corona graphs $(G \circ H)$ and we solve the power domination number for splitting graph of corona graph. Let $G$ be a graph of order $r \geq 2$ and $H$ be a graph of order $s \geq 2$. Then $\gamma_{p}(S(G \circ H))=r$.

Proof. Label the vertices of $G$ as $v_{1}, v_{2}, \ldots, v_{r}$ and let $u_{1}, u_{2}, \ldots, u_{s}$ be the vertices of $H$ which are replicated by the vertices $v_{1}^{\prime}, v_{2}^{\prime}, \ldots, v_{r}^{\prime}$ and $u_{1}^{\prime}, u_{2}^{\prime}, \ldots, u_{s}^{\prime}$. Then the graph $S(G \circ H)$ will have $2(r+r s)$ vertices. Let $S$ be a power dominating set of $S(G \circ H)$. Suppose not, by definition, there are $r$ vertex disjoint copies of $H_{k}, 1 \leq k \leq r$ adjacent to each vertex of $G$ in $S(G \circ H)$. With the deletion of a vertex say, $k^{t h}$ vertex from $S$ we get that $k^{t h}$ copy of $H$ and replicated vertices of $k^{t h}$ copy of $H$ not monitored by $S$, 
a contradiction. Thus $|S| \geq r$. Now let $S=\left\{v_{1}, v_{2}, \ldots, v_{r}\right\}=V(S(G \circ H))$. Hence $M^{0}(S)=V(G)$ is monitored by $S$. Therefore, $\gamma_{p}(S(G \circ H))=r$. Hence the proof.

\section{Degree Splitting Graph}

In this section, we compute the power domination number for degree splitting graph .

Basavanagoudi et al. [11] have given a lower bound and upper bound for the domination number of degree splitting graph for any graph $G$ and is quoted below.

[11] For any graph $G, 1 \leq \gamma(D S(G)) \leq\left|w_{i} \cup L\right|$, for every $w_{i}, 1 \leq i \leq r$.

\section{Observations:}

The following results hold good:

$a$. For any graph $G, 1 \leq \gamma_{p}(D S(G)) \leq\left|w_{i} \cup L\right|$, for every $w_{i}, 1 \leq i \leq r$.

b. For $n \geq 3, \gamma_{p}(D S(G))=1, G \in\left\{P_{n}, F_{m}, W_{m}, H_{m}, K_{1, n}, K_{m, n}, P_{m} P_{n}, P_{m} C_{n}\right.$, $P_{n} K_{m}, P_{n} K_{1, m}, P_{n} W_{m}, C_{n} K_{m}, C_{n} K_{1, n}, C_{n} W_{m}, W_{n} W_{m}, W_{n} K_{1, m}, K_{n} K_{m}, P_{m} \times$ $\left.P_{n}, P_{m} \times C_{n}\right\}$. See Figure 3(a).

$c$. Let $G$ be a regular graph of order $n \geq 3$. Then $\gamma_{p}(D S(G))=1$.

$d$. Let $G$ be a spider tree $S(n)$. Then $\gamma_{p}(S(G))=1$.

$e$. For any graph $G, \gamma_{p}(D S(G))=\gamma_{p}(G), G \in\left\{P_{n}, C_{n}, F_{n}, W_{n}, K_{n}, K_{1, n}, K_{m, n}, S(n)\right\}$, $m \leq n, m, n \geq 3$.

[8] Let $T_{r}$ be the triangular grid graph. The vertices of $T_{r}$ are ordered triples $(i, j, k)$ of non-negative integers summing to $r$, and two vertices are connected by an edge if they admit in one co-ordinate and vary by one in the other two co-ordinates. Vertices in a $r$-dimensional hexagonal graph are placed at the vertices of a $r$-triangular tessellation, so that each vertx has up to $2 k+2$ adjacent neighbours, denoted by $H X(r)$. See Figure $3(b)$. 


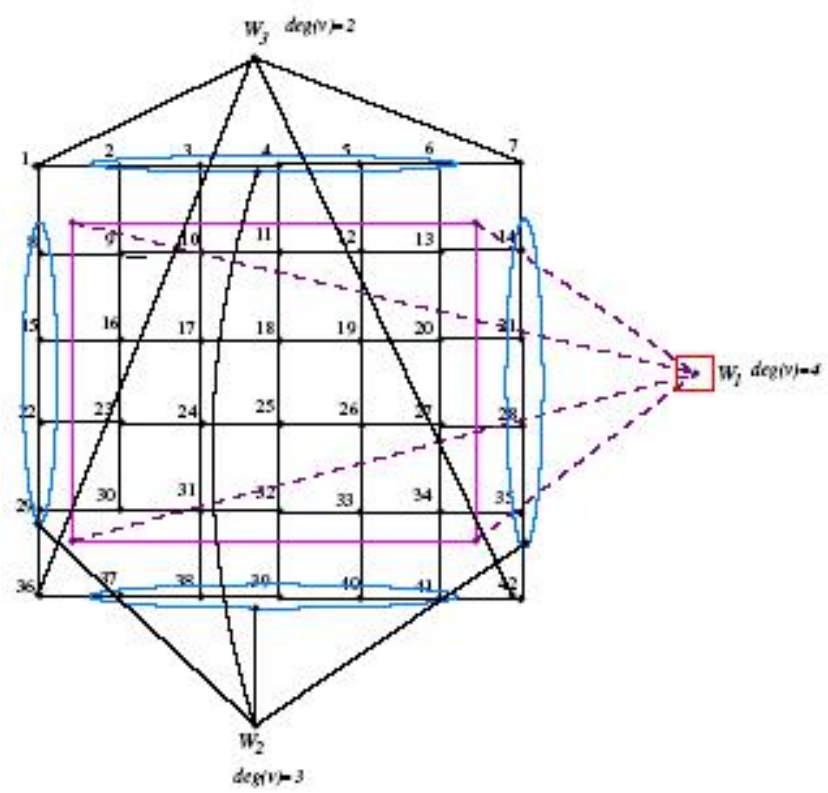

(a)

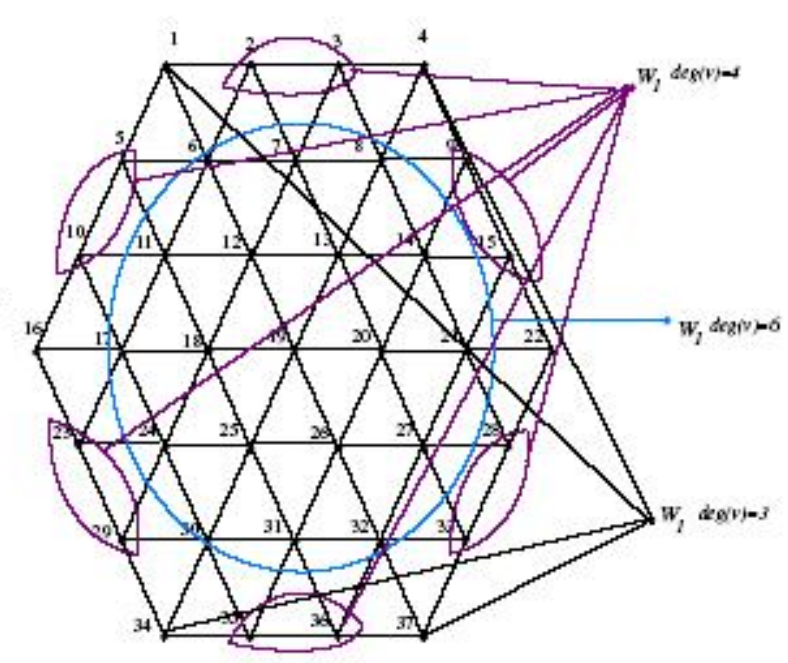

(b)

Figure 3.1: Red colored squared vertices indicates a minimum power dominating set of (a) $P_{6} P_{7} \quad(b)$ Hexagonal grid $H X(3)$.

Let $G$ be a Hexagonal grid $H X(r), n \geq 2$ or triangular grid $T(r), n \geq 2$. Then $\gamma_{p}(D S(G))=2$.

Proof. Let $S$ be a power dominating set of $D S(H X(r))$ with $|S|=2$. Suppose not, let $|S|<2$.

Case (i): Let $S=\{w: \operatorname{deg}(w)=3\}$. Then for every vertex in $v \in N(w)$ is adjacent to two unmonitored vertices of degree four, a contradiction. Therefore, $|S| \neq 1$. Thus $|S| \geq 2$.

Case (ii): Let $S=\{w: \operatorname{deg}(w)=4\}$. Then at least one vertex in $u \in N(w)$ is adjacent to two unmonitored vertices, a contradiction. 
Case (iii): Let $S=\{w: \operatorname{deg}(w)=6\}$. Then at least one vertex in $u \in N(w)$ is adjacent to two unmonitored vertices, a contradiction.

$(\leq:)$ Let $S=\left\{w_{1}, w_{2}\right\}$. Then $S$ monitor all vertices of degree 4 and 6 . Now every vertex in $M^{0}(S)$ is adjacent to exactly one unmonitored vertices of degree 3. These vertex monitor in $M^{1}(S)$. Now vertex $w_{3}$ monitor in $M^{2}(S)$. Hence $M^{2}(S)=V(D S(G))$ is monitored by $S$. Therefore, $\gamma_{p}(D S(G))=2$. Hence the proof.

Observation 3. For the complete $k$ - array tree $T$ of level $r, r \geq 2, k \geq$ $2, \gamma_{p}(D S(T))=2$.

\section{Conclusion and Open Problem}

In this paper, we have obtained the power domination numbers for splitting graph and degree splitting graph. Further, we realized partial solutions of graphs for which $\gamma_{p}(S(G))=\gamma_{p}(G), \gamma_{p}(S(G))=\gamma_{p}(G)=\gamma(G)$ and $\gamma_{p}(D S(G))=\gamma_{p}(G)$. Further, identifying classes of graphs for which $\gamma_{p}(S(G))=\gamma_{p}(G)=\gamma(G)$ is open.

\section{References}

[1] T. W. Haynes, S. M. Hedetniemi, S. T. Hedetniemi, and M. A. Henning, "Power domination in graphs applied to electrical power networks", SIAM journal on discrete mathematics, vol. 15, no. 4, pp. 519-529, 2002.

[2] G. J. Chang, P. Dorbec, M. Montassier, and A. Raspud, "Generalized power domination of graphs", Discrete applied mathematics, vol. 160, no.12, pp. 1691-1698, 2012.

[3] K. F. Benson, D.Ferrero, M. Flagg, V. Furst, L. Hogben, V. Vasilevskak, and B. Wissman, "Zero forcing and power domination for graph products", 2017, arXiv: 1510.02421.

[4] G. J. Xu, L.Y. Kang, E. F. Shan, and M. Zhao, "Power domination in block graphs”, Theoretical computer science, vol. 359, pp. 299-305, 2006.

[5] P. Dorbec, M. Mollard, S. Klavzar, and S. Spacapan, "Power domination in product graphs", SIAM journal on discrete mathematics, vol. 22, no. 2, pp. 554-567, 2008. 
[6] R. Barrera and D. Ferrero, "Power domination in cylinders, tori, and the generalized Petersen graphs”, Networks, pp. 43-49, 2009.

[7] S. Stephen, B. Rajan, J. Ryan, and C. Grigorious, "Power domination in certain chemical structures", Journal of discrete algorithms, vol. 33, pp. 10-18, 2015.

[8] P. Bose, C. Pennarun, and S. Verdonschot, "Power domination on triangular grids", 2017, arXiv:1707.02760.

[9] F. Kazemmnejad and S. Moradi, "Total domination number of central graph", Bulletin of the Korean Mathematical Society, vol. 56, no. 4, pp. 1059-1075, 2019.

[10] J. Anitha, "2-Power domination number 1 for certain interconnection networks", International journal applied engineering research, vol. 109, no. 5, pp. 59-66, 2016.

[11] B. Basavanagoudi, P. V. Patil, and S. M. Hosamani, "Domination in degree splitting graphs", Journal of analysis and computation, vol. 10, no. 34, pp. 27336-27341, 2015.

[12] J. Anitha and I. Rajasingh , "Power domination parameters in hypermesh-pyramid networks and corona graphs", International journal of pure and applied mathematics, vol. 109, no. 5, pp. 59-66, 2016.

[13] R. Sundara Rajan, J. Anitha, and I. Rajasingh, "2- Power domination in certain interconnection networks", Procedia computer science, vol. 57, pp. 738-744, 2015.

[14] M. Miller, R. S. Rajan, R. Jayagopal, I. Rajasingh, and P. Manuel, "A note on the locating-total domination in graphs", Discussiones mathematicae graph theory, vol. 37, pp. 745-754, 2017 
J. Anitha

Department of Mathematics

Arulmigu Kapaleeswarar Arts and

Science College

Chennai-99,

India

e-mail: anithaharish78@gmail.com

Corresponding Author

and

S. Muthukumar

Department of Mathematics

Easwari Engineering College

Chennai 600 089,

India

e-mail: hod.maths@eec.srmrmp.edu.in 